\title{
Contribution of XPD and XPF Polymorphisms to Susceptibility of Non-Small Cell Lung Cancer in High-Altitude Areas
}

\author{
Miao Li ${ }^{\mathrm{a}}$ Rong Chen ${ }^{\mathrm{b}}$ Baoyan $\mathrm{Ji}^{\mathrm{c}}$ Chunmei Fan ${ }^{\mathrm{d}}$ Guanying Wang ${ }^{\mathrm{e}}$ \\ Chenli Yue ${ }^{f}$ Guoquan Li ${ }^{a}$ \\ aDepartment of Medicine Oncology, The Fifth People's Hospital of Qinghai Province, Xining, China; ${ }^{b}$ Department \\ of Medicine Oncology, The Affiliated Hospital of Qinghai University, Xining, China; 'Department of Medicine \\ Oncology, The People's Hospital of Qinghai Province, Xining, China; ${ }^{\mathrm{d} D e p a r t m e n t}$ of Science and education, \\ The Fifth People's Hospital of Qinghai Province, Xining, China; eDepartment of Oncology, The Second Affiliated

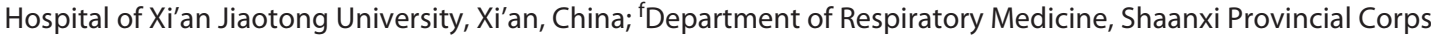 \\ Hospital of Chinese People's Armed Police Force, Xi'an, China
}

\section{Keywords}

Non-small cell lung cancer - XPD - XPF · Susceptibility ·

Cisplatin-based chemotherapy

\begin{abstract}
Background: We aimed to explore the relation of XPD and $X P F$ variants with non-small cell lung cancer (NSCLC) risk and the effect of these variants on the sensitivity to cisplatinbased chemotherapy among the Chinese Han population in high-altitude areas. Methods: Eight single-nucleotide polymorphisms (SNPs) in XPD and XPF were genotyped by Agena MassARRAY platform among 506 NSCLC cases and 510 healthy controls. Correlation of XPD and XPF gene polymorphisms with NSCLC susceptibility and the response of cisplatin-based chemotherapy were analyzed with logistic regression by calculating odds ratios (ORs) and 95\% confidence intervals (Cls). Results: XPD rs13181 (OR $=1.53,95 \%$ $\mathrm{Cl}: 1.04-2.24, p=0.029)$ and $\mathrm{rs} 1052555(\mathrm{OR}=1.63,95 \% \mathrm{Cl}$ : $1.05-2.53, p=0.029)$ possibly contributed to the increased risk of lung adenocarcinoma, while XPD rs238406 (OR = 0.63,
\end{abstract}

95\% Cl: $0.43-0.94, p=0.024)$ was a protective factor for lung squamous cell carcinoma. Age, gender, BMI, smoking, and drinking might affect the correlation of XPD and XPF polymorphisms with NSCLC risk. More importantly, XPD rs13181 $(\mathrm{OR}=2.91, p=0.015), X P D$ rs1052555 (OR $=2.67, p=0.022)$, and $X P F$ rs231127 (OR $=4.15, p=0.008)$ were associated with treatment response in NSCLC patients underwent cisplatinbased chemotherapy. Conclusion: This study found that $X P D$ and $X P F$ variants might contribute to NSCLC risk and the response of cisplatin-based chemotherapy among the Chinese Han population in high-altitude areas.

(c) 2021 S. Karger AG, Basel

\section{Introduction}

There were an estimated 2.1 million new cases and 1.8 million deaths for lung cancer worldwide in 2018, which accounted for close to 1 in 5 (18.4\%) cancer deaths [1]. In China, lung cancer is the most commonly diagnosed and was identified as the leading cause of cancer death $(17.1 \%$ karger@karger.com www.karger.com/phg

(C) 2021 S. Karger AG, Base

Karger!
Correspondence to:

Guoquan Li, guoquanli0321@163.com 
for incidence and $21.7 \%$ for mortality, respectively) [2]. Among them, two-thirds of lung cancer deaths occur in males and about $55 \%$ of which are due to active smoking. Other factors such as alcohol, poor diet, occupational exposures, and air pollution may also had effect on the occurrence and development of lung cancer, especially nonsmall cell lung cancer (NSCLC) [3]. Existing studies have revealed the strong association between genetic variations and the susceptibility to NSCLC $[4,5]$. Moreover, genetic variations are considered as important factors to influence the treatment efficacy and prognosis of NSCLC. Interindividual variability in response to cisplatin for NSCLC patients was associated with genetic variants [6-8].

Nucleotide excision repair (NER) participates in DNA repair pathways which were involved in the repair of chromosomal breakage or rearrangement in the processes of DNA damage, replication errors, and recombination [9]. Xeroderma pigmentosum complementation group D (XPD) and xeroderma pigmentosum complementation group F (XPF) are important components of NER. XPD (also called excision repair cross-complimentary group 2), an ATP-dependent helicase, is an essential member of the basal transcription factor TFIIH complex for normal transcription initiation of NER [10].XPF, also called excision repair cross-complimentary group 4 , involves in the recombination repair, mismatch repair, and identification of damage sites [11]. Saviozzi et al. [12] reported that $X P D$ and $X P F$ genes were significantly overexpressed in NSCLC tissue compared with normal lung tissue. Previous studies have reported that XPD and XPF polymorphisms were associated with the susceptibility and response to chemotherapy of cancers, including NSCLC. However, those currently available results have been inconsistent $[13,14]$.

Qinghai-Tibet Plateau is located in northwest China, at an average altitude of over 3,000 m. In high-altitude areas, perennial hypobaric hypoxia and strong ultraviolet radiation may cause genetic variation, which, in turn, affects the occurrence of diseases including cancers. The occurrence and development of NSCLC in high-altitude areas have been fewly reported. The Han population is the largest ethnic group in China and is also widely distributed in high-altitude areas. Here, our study investigated the correlation between $X P D$ and $X P F$ variants and NSCLC risk among the Chinese Han population in highaltitude areas and assessed the effect of variants on the sensitivity of NSCLC patients to cisplatin-based chemotherapy.

\section{Materials and Methods}

\section{Subjects}

This study included 1,016 subjects comprised 506 NSCLC cases ( $59.80 \pm 10.63$ years, 350 males and 156 females) and 510 healthy controls (59.80 \pm 9.08 years, 353 males and 157 females). All subjects were genetically unrelated ethnic Han Chinese. All patients were diagnosed and histopathologically confirmed primary NSCLC from the Qinghai Province Cancer Hospital. Healthy controls were enrolled from the health checkup center of the same hospital. The patients had no history of cancer. Blood samples of patients were gathered before beginning chemotherapy or radiotherapy. The exclusion criteria of the control group included chronic respiratory disease, tuberculosis, inflammation, and autoimmune disorders. Epidemiological and clinical data were available from medical records and standardized questionnaires, respectively. Data on basic information including age, gender, BMI, smoking, and drinking exposure and clinical information about pathological types, lymph nodes metastasis, and stages were collected.

Evaluation of Cisplatin-Based Chemotherapeutic Response

One hundred and eighty-nine NSCLC patients who received cisplatin-based chemotherapy satisfied the Eastern Cooperative Oncology Group performance status 0 or 1 . These patients received cisplatin-based chemotherapy every 3 weeks until 6 cycles or tumor progression. Adverse effects were assessed according to the National Cancer Institute Common Toxicity Criteria, version 3.0 standard [15]. The patients were subdivided into toxicity and nontoxicity group. For response to therapy, patients with complete response (CR) or partial response (PR) were defined as the " $\mathrm{CR}+$ $\mathrm{PR}^{\prime}$ group, and patients achieving stable disease (SD) or progressive disease $(\mathrm{PD})$ were considered as the "SD + PD" group according to the Response Evaluation Criteria in Solid Tumor Group (RECIST) guidelines [16].

Single-Nucleotide Polymorphisms Selection and Genotyping

The candidate SNPs in XPD and XPF were selected based on previously reported genetic variants related to NSCLC susceptibility [17]. Of those, we identified tagged SNPs with $>5 \%$ minor allele frequency for Chinese in the 1,000 Genomes Project data (http:// www.internationalgenome.org/), and the linkage disequilibrium of a pairwise tagging $r^{2} \geq 0.8$. Finally, 8 SNPs in XPD (rs13181, rs1052555, rs238406, and rs1799793) and XPF (rs231127, rs8054265, rs6498486, and rs3743538) were selected for further genotyping. Peripheral venous blood $(5 \mathrm{~mL})$ was drawn from each subject in the EDTA-coated tubes and stored at $-80^{\circ} \mathrm{C}$ after centrifugation. GoldMag DNA Purification Kit (GoldMag Co., Ltd., $\mathrm{Xi}^{\prime}$ an City, China) was used to extract gDNA, and the concentration and purity of the extracted DNA were determined by NanoDrop 2000. We used the Agena MassARRAY platform for SNPs genotyping in accordance with the manufacturer's protocol in a double-blind fashion by 2 people. $5 \%$ of samples were randomly selected for re-genotyping. Call rates of genotyping were $>95 \%$, and the concordance rate reached $100 \%$.

\section{Statistical Analysis}

SPSS 18.0 (SPSS Inc., Chicago, IL, USA) and PLINK 1.0.7 were performed. To assess the differences of demographic and clinical data between the patients and the controls, $\chi^{2}$ tests for categorical 
variables and independent sample $t$ test for continuous variables were carried out. Hardy-Weinberg equilibrium in controls was evaluated by the goodness-of-fit $\chi^{2}$ tests. The correlation of XPD and $X P F$ variants with NSCLC risk was analyzed using logistic regression by odds ratios (ORs) and 95\% confidence intervals (CIs). Further, we performed stratified analysis by gender, age, BMI, status of smoking and drinking, histology, stage, and lymph nodes metastasis. Linkage disequilibrium and haplotype analyses were calculated by Haploview software (version 4.2) and PLINK software. Logistic regression method was utilized to analyze the correlation between SNPs and the effectiveness of cisplatin-based chemotherapy in NSCLC patients. Two-sided $p$ value $<0.05$ was examined statistically significant.

\section{Results}

\section{Study Participants}

The characteristics of the subjects were shown in Table 1 . There were no significant differences in age $(p=$ $0.351)$ and gender $(p=0.987)$ distribution between NSCLC patients and healthy individuals. Table 1 also gave the proportion of BMI, smoking and drinking exposures of all participants, and histological types, lymph nodes metastasis, and stages of patients. The analysis included 189 NSCLC patients treated with cisplatin-based chemotherapy.

\section{The Correlation of XPD and XPF Polymorphisms with} NSCLC Risk

Eight SNPs within $X P D$ and $X P F$ were genotyped among NSCLC patients and healthy controls. The minor allele frequency of the selected SNPs was $>5 \%$ (online suppl. Table 1; for all online suppl. material, see www. karger.com/doi/10.1159/000512641). And the genotype of all SNPs in controls was consistent with Hardy-Weinberg equilibrium $(p>0.05)$, which indicated the subjects had a representative of the population. The genotype and allele frequencies of NSCLC cases and controls were revealed in online suppl. Table 2. However, there was no significant difference in genotype and allele frequencies of any SNPs between cases and controls $(p>0.05)$.

The Effect of Age, Gender, BMI, Smoking, and

Drinking on the Correlation of XPD and XPF

Variants with NSCLC Risk

The effects of age, gender, BMI, smoking, and drinking on the correlation of XPD and XPF variants with NSCLC risk were evaluated (Tables 2,3 ). In the stratified analysis by age (Table 2), rs13181-G allele increased NSCLC susceptibility at age $>59$ years in the allele $(\mathrm{OR}=1.63,95 \%$ CI: $1.03-2.57, p=0.035)$, dominant $(\mathrm{OR}=1.62,95 \% \mathrm{CI}$ :
Table 1. Characteristics of NSCLC patients and health controls

\begin{tabular}{|c|c|c|c|}
\hline Variables & $\begin{array}{l}\text { Cases } \\
(n=506)\end{array}$ & $\begin{array}{l}\text { Control } \\
(n=510)\end{array}$ & $\begin{array}{l}p \\
\text { value }\end{array}$ \\
\hline \multicolumn{4}{|l|}{ Age, $n(\%)$} \\
\hline Mean $\pm S D$, years & $59.80 \pm 10.63$ & $59.80 \pm 9.08$ & \multirow{3}{*}{0.351} \\
\hline$>59$ years & $271(53.6)$ & $275(53.9)$ & \\
\hline$\leq 59$ years & $235(46.4)$ & $235(46.1)$ & \\
\hline \multicolumn{4}{|l|}{ Gender, $n(\%)$} \\
\hline Male & $350(69.2)$ & $353(69.2)$ & \multirow{2}{*}{0.987} \\
\hline Female & $156(30.8)$ & $157(30.8)$ & \\
\hline \multicolumn{4}{|l|}{$B M I, n(\%)$} \\
\hline$\leq 24 \mathrm{~kg} / \mathrm{m}^{2}$ & $133(26.3)$ & $138(27.1)$ & \\
\hline$>24 \mathrm{~kg} / \mathrm{m}^{2}$ & $81(16.0)$ & $181(35.5)$ & \\
\hline Unavailable & $292(57.7)$ & $191(37.5)$ & \\
\hline \multicolumn{4}{|l|}{ Smoking, $n(\%)$} \\
\hline Yes & $242(47.8)$ & $108(21.2)$ & \\
\hline No & $161(31.8)$ & $180(35.3)$ & \\
\hline Unavailable & $103(20.4)$ & $222(43.5)$ & \\
\hline \multicolumn{4}{|l|}{ Drinking, $n(\%)$} \\
\hline Yes & $109(21.5)$ & $103(20.2)$ & \\
\hline No & $267(52.8)$ & $156(30.6)$ & \\
\hline Unavailable & $130(25.7)$ & $251(49.2)$ & \\
\hline \multicolumn{4}{|l|}{ Pathological type, $n(\%)$} \\
\hline Squamous carcinoma & $174(34.4)$ & & \\
\hline Adenocarcinoma & $212(41.9)$ & & \\
\hline Unavailable & $120(23.7)$ & & \\
\hline \multicolumn{4}{|c|}{ Lymph node metastasis, $n(\%)$} \\
\hline Yes & $269(53.2)$ & & \\
\hline No & $103(20.4)$ & & \\
\hline Unavailable & $134(26.5)$ & & \\
\hline
\end{tabular}

\begin{tabular}{lr}
\hline Stage, $n(\%)$ & \\
I-II & $93(18.4)$ \\
III-IV & $286(56.5)$ \\
Unavailable & $127(25.1)$ \\
\hline Cisplatin-based chemotherapy $(n=189)$ \\
Toxicity & $37(19.6)$ \\
Nontoxicity & $152(80.4)$ \\
CR + PR & $42(22.2)$ \\
SD + PD & $100(52.9)$ \\
Unavailable & $47(24.9)$
\end{tabular}

NSCLC, non-small cell lung cancer; SD, standard deviation; $\mathrm{CR}$, complete response; $\mathrm{PR}$, partial response; $\mathrm{SD}$, stable disease; $\mathrm{PD}$, progression of disease.

$1.00-2.62, p=0.049)$, and additive models $(\mathrm{OR}=1.64$, $95 \%$ CI: $1.02-2.63, p=0.040)$. The rs1052555-A allele also conferred a higher NSCLC risk at age $>59$ years in the allele $(\mathrm{OR}=1.99,95 \% \mathrm{CI}: 1.18-3.35, p=0.008)$, heterozygote $(\mathrm{OR}=1.87,95 \% \mathrm{CI}: 1.08-3.24, p=0.024)$, dominant $(\mathrm{OR}=1.92,95 \% \mathrm{CI}: 1.12-3.32, p=0.019)$, and additive 
Table 2. Relationships between XPD polymorphisms and NSCLC risk according to the stratification by age and gender

\begin{tabular}{|c|c|c|c|c|c|}
\hline SNP id & Models & OR (95\% CI) & $p$ value & OR (95\% CI) & $p$ value \\
\hline Age & & $>59$ years & & $\leq 59$ years & \\
\hline \multirow[t]{6}{*}{ rs13181 } & Allele & $1.63(1.03-2.57)$ & 0.035 & $0.98(0.63-1.52)$ & 0.910 \\
\hline & Heterozygote & $1.59(0.98-2.57)$ & 0.060 & $1.04(0.64-1.68)$ & 0.880 \\
\hline & Homozygote & - & - & - & - \\
\hline & Dominant & $1.62(1.00-2.62)$ & 0.049 & $0.99(0.61-1.59)$ & 0.956 \\
\hline & Recessive & - & - & - & - \\
\hline & Log-additive & $1.64(1.02-2.63)$ & 0.040 & $0.93(0.59-1.47)$ & 0.766 \\
\hline \multirow[t]{6}{*}{ rs 1052555} & Allele & $1.99(1.18-3.35)$ & 0.008 & $0.97(0.57-1.62)$ & 0.895 \\
\hline & Heterozygote & $1.87(1.08-3.24)$ & 0.024 & $1.01(0.58-1.75)$ & 0.978 \\
\hline & Homozygote & - & - & - & - \\
\hline & Dominant & $1.92(1.12-3.32)$ & 0.019 & $0.97(0.56-1.68)$ & 0.916 \\
\hline & Recessive & - & - & - & - \\
\hline & Log-additive & $1.94(1.14-3.32)$ & 0.015 & $0.93(0.55-1.59)$ & 0.800 \\
\hline Gender & & Males & & Females & \\
\hline \multirow[t]{6}{*}{ rs238406 } & Allele & $0.81(0.66-1.01)$ & 0.056 & $1.12(0.82-1.54)$ & 0.474 \\
\hline & Heterozygote & $0.64(0.46-0.90)$ & 0.011 & $1.43(0.85-2.41)$ & 0.182 \\
\hline & Homozygote & $0.72(0.48-1.09)$ & 0.122 & $1.21(0.65-2.26)$ & 0.542 \\
\hline & Dominant & $0.67(0.49-0.92)$ & 0.012 & $1.36(0.83-2.21)$ & 0.225 \\
\hline & Recessive & $0.93(0.65-1.34)$ & 0.699 & $0.97(0.57-1.65)$ & 0.917 \\
\hline & Log-additive & $0.82(0.67-1.01)$ & 0.065 & $1.12(0.82-1.52)$ & 0.479 \\
\hline
\end{tabular}

NSCLC, non-small cell lung cancer; SNP, single-nucleotide polymorphism; OR, odds ratio; CI, confidence interval. $p$ values were calculated by logistic regression analysis with adjustments for age and gender. Bold $p<$ 0.05 means the data are statistically significant. “-” represents the $p$ values were unavailable due to the absence of frequency of genotype.

models $(\mathrm{OR}=1.94,95 \%$ CI: $1.14-3.32, p=0.015)$. Stratified by gender (Table 2), rs238406 was a protective factor for NSCLC occurrence in males (heterozygote: $\mathrm{OR}=0.64$, 95\% CI: 0.46-0.90, $p=0.011$; and dominant: $\mathrm{OR}=0.67$, 95\% CI: $0.49-0.92, p=0.012)$. In the BMI stratification (Table 3), rs3743538 (TT vs. GG, OR = 3.81, 95\% CI: $1.08-13.45, p=0.038$ and TT vs. GG-GT, OR $=3.53,95 \%$ CI: $1.01-12.26, p=0.048)$ increased NSCLC risk in the subgroup with BMI $>24 \mathrm{~kg} / \mathrm{m}^{2}$. Among the subgroup with BMI $\leq 24 \mathrm{~kg} / \mathrm{m}^{2}$, rs231127 (OR $=0.38,95 \%$ CI: $0.15-$ $0.94, p=0.036)$ and $\mathrm{rs} 6498486(\mathrm{OR}=1.72,95 \% \mathrm{CI}: 1.02$ $2.90, p=0.042)$ were related to NSCLC susceptibility. In smokers, the rs $238406-\mathrm{T}$ allele ( $\mathrm{T}$ vs. $\mathrm{G}, \mathrm{OR}=1.51,95 \%$ CI: $1.09-2.09, p=0.012$ and additive, $\mathrm{OR}=1.51,95 \% \mathrm{CI}$ : $1.09-2.10, p=0.014$ ) and TT genotype (TT vs. GG, OR = $2.15,95 \%$ CI: $1.06-4.37, p=0.035$ and TT vs. GG-GT, $\mathrm{OR}=2.66,95 \% \mathrm{CI}: 1.53-4.63, p=0.001)$ had the increased NSCLC susceptibility (Table 3). Among drinkers, rs13181 (TG vs. TT, $\mathrm{OR}=2.25,95 \% \mathrm{CI}: 1.03-4.94, p=0.043$ ) contributed to the increased risk of NSCLC, while rs238406 (GT vs. GG, $\mathrm{OR}=0.33,95 \% \mathrm{CI}: 0.17-0.62, p=0.001$ and GT-TT vs. GG, OR $=0.45,95 \%$ CI: $0.25-0.81, p=0.008$ ) decreased the risk (Table 3 ). 
Table 3. Relationships between XPD and XPF polymorphisms and NSCLC risk according to the stratification by $\mathrm{BMI}$ and the status of smoking and drinking

\begin{tabular}{|c|c|c|c|c|c|}
\hline SNP id & Models & OR (95\% CI) & $p$ value & OR (95\% CI) & $p$ value \\
\hline BMI & & $>24 \mathrm{~kg} / \mathrm{m}^{2}$ & & $\leq 24 \mathrm{~kg} / \mathrm{m}^{2}$ & \\
\hline \multirow[t]{6}{*}{ rs231127 } & Allele & $1.18(0.80-1.75)$ & 0.393 & $0.84(0.59-1.20)$ & 0.339 \\
\hline & Heterozygote & $1.03(0.59-1.82)$ & 0.909 & $1.15(0.69-1.90)$ & 0.602 \\
\hline & Homozygote & $1.75(0.69-4.47)$ & 0.239 & $0.40(0.16-1.05)$ & 0.063 \\
\hline & Dominant & $1.13(0.65-1.94)$ & 0.665 & $0.98(0.60-1.60)$ & 0.936 \\
\hline & Recessive & $1.72(0.71-4.17)$ & 0.227 & $0.38(0.15-0.94)$ & 0.036 \\
\hline & Log-additive & $1.21(0.79-1.84)$ & 0.375 & $0.82(0.56-1.19)$ & 0.295 \\
\hline \multirow[t]{6}{*}{ rs6498486 } & Allele & $1.14(0.73-1.76)$ & 0.569 & $1.37(0.90-2.06)$ & 0.139 \\
\hline & Heterozygote & $1.24(0.71-2.16)$ & 0.461 & $1.72(1.02-2.90)$ & 0.042 \\
\hline & Homozygote & $1.38(0.39-4.91)$ & 0.617 & $1.02(0.32-3.19)$ & 0.976 \\
\hline & Dominant & $1.25(0.73-2.15)$ & 0.419 & $1.61(0.98-2.65)$ & 0.062 \\
\hline & Recessive & $1.27(0.37-4.43)$ & 0.705 & $0.84(0.27-2.60)$ & 0.766 \\
\hline & Log-additive & $1.21(0.77-1.90)$ & 0.413 & $1.36(0.90-2.06)$ & 0.149 \\
\hline \multirow[t]{6}{*}{ rs3743538 } & Allele & $1.46(0.92-2.31)$ & 0.104 & $1.34(0.87-2.07)$ & 0.190 \\
\hline & Heterozygote & $1.27(0.70-2.29)$ & 0.430 & $1.38(0.80-2.37)$ & 0.244 \\
\hline & Homozygote & $3.81(1.08-13.45)$ & 0.038 & $1.46(0.44-4.82)$ & 0.534 \\
\hline & Dominant & $1.47(0.84-2.56)$ & 0.180 & $1.39(0.83-2.33)$ & 0.210 \\
\hline & Recessive & $3.53(1.01-12.26)$ & 0.048 & $1.33(0.41-4.35)$ & 0.636 \\
\hline & Log-additive & $1.55(0.97-2.45)$ & 0.065 & $1.30(0.85-2.00)$ & 0.228 \\
\hline Smoking & & Yes & & No & \\
\hline \multirow[t]{6}{*}{ rs238406 } & Allele & $1.51(1.09-2.09)$ & 0.012 & $1.06(0.78-1.43)$ & 0.709 \\
\hline & Heterozygote & $0.74(0.41-1.36)$ & 0.335 & $1.02(0.62-1.70)$ & 0.929 \\
\hline & Homozygote & $2.15(1.06-4.37)$ & 0.035 & $0.97(0.52-1.81)$ & 0.918 \\
\hline & Dominant & $1.07(0.60-1.91)$ & 0.811 & $1.01(0.63-1.62)$ & 0.981 \\
\hline & Recessive & $2.66(1.53-4.63)$ & 0.001 & $0.95(0.55-1.65)$ & 0.868 \\
\hline & Log-additive & $1.51(1.09-2.10)$ & 0.014 & $0.99(0.73-1.35)$ & 0.937 \\
\hline
\end{tabular}

(Table continued on next page.)

Relationship of Clinicopathological Features with XPD and XPF Variants

Further, the correlation between XPD and XPF SNPs and clinicopathological features was assessed, including histological subgroups, clinical stage (III-IV vs. I-II), and lymphatic metastatic status (metastasis vs. nonmetastasis). The results of stratified analyses by histology types showed in Table 4. In lung adenocarcinoma, the allele and/or gen- otype frequencies of rs13181 and rs1052555 were significantly different. Rs13181 (Gvs. T, OR $=1.53,95 \%$ CI: $1.04-$ 2.24, $p=0.029$; TG vs. TT, OR $=1.70,95 \%$ CI: $1.13-2.57$, $p=0.011 ;$ and TG-GG vs. TT, OR $=1.65,95 \%$ CI: 1.10 $2.49, p=0.016$ ) and $\mathrm{rs} 1052555$ (A vs. $\mathrm{G}, \mathrm{OR}=1.63,95 \% \mathrm{CI}$ : $1.05-2.53, p=0.029$; GA vs. GG, OR $=1.75,95 \%$ CI: 1.10 $2.79, p=0.019$; and GA-AA vs. $\mathrm{GG}, \mathrm{OR}=1.71,95 \% \mathrm{CI}$ : $1.07-2.71, p=0.024)$ were related to the increased risk of 
Table 3 (continued)

\begin{tabular}{|c|c|c|c|c|c|}
\hline \multicolumn{2}{|l|}{ Drinking } & \multicolumn{2}{|l|}{ Yes } & \multicolumn{2}{|l|}{ No } \\
\hline \multirow[t]{6}{*}{ rs13181 } & Allele & $1.75(0.86-3.56)$ & 0.118 & $1.09(0.66-1.79)$ & 0.745 \\
\hline & Heterozygote & $2.25(1.03-4.94)$ & 0.043 & $1.07(0.63-1.82)$ & 0.807 \\
\hline & Homozygote & - & - & - & - \\
\hline & Dominant & $2.07(0.96-4.46)$ & 0.064 & $1.09(0.64-1.86)$ & 0.741 \\
\hline & Recessive & - & - & - & - \\
\hline & Log-additive & $1.82(0.87-3.78)$ & 0.110 & $1.12(0.67-1.89)$ & 0.666 \\
\hline \multirow[t]{6}{*}{ rs238406 } & Allele & $0.82(0.56-1.21)$ & 0.314 & $1.00(0.76-1.33)$ & 0.978 \\
\hline & Heterozygote & $0.33(0.17-0.62)$ & 0.001 & $0.98(0.62-1.54)$ & 0.931 \\
\hline & Homozygote & $0.88(0.41-1.93)$ & 0.757 & $1.04(0.58-1.85)$ & 0.906 \\
\hline & Dominant & $0.45(0.25-0.81)$ & 0.008 & $0.99(0.65-1.53)$ & 0.981 \\
\hline & Recessive & $1.69(0.86-3.33)$ & 0.128 & $1.05(0.63-1.74)$ & 0.855 \\
\hline & Log-additive & $0.85(0.58-1.23)$ & 0.391 & $1.01(0.76-1.35)$ & 0.930 \\
\hline
\end{tabular}

NSCLC, non-small cell lung cancer; SNP, single-nucleotide polymorphism; OR, odds ratio; CI, confidence interval. $p$ values were calculated by logistic regression analysis with adjustments for age and gender. Bold $p<$ 0.05 means the data are statistically significant. "-" represents the $p$ values were unavailable due to the absence of frequency of genotype.

Table 4. The association of XPD polymorphisms and NSCLC susceptibility

\begin{tabular}{|c|c|c|c|c|c|c|c|c|}
\hline \multirow[t]{2}{*}{ SNP id } & \multirow[t]{2}{*}{ Genotype } & \multirow[t]{2}{*}{ Control } & \multicolumn{3}{|c|}{ Adenocarcinoma } & \multicolumn{3}{|c|}{ Squamous cell carcinoma } \\
\hline & & & case & OR (95\% CI) & $p$ value & case & OR (95\% CI) & $p$ value \\
\hline \multirow{3}{*}{ rs13181 } & G & 77 & 47 & $1.53(1.04-2.24)$ & 0.029 & 25 & $0.95(0.59-1.51)$ & 0.823 \\
\hline & TT & 435 & 165 & 1 & & 149 & 1 & \\
\hline & TG & 73 & 47 & $1.70(1.13-2.57)$ & 0.011 & 25 & $1.05(0.64-1.74)$ & 0.840 \\
\hline \multirow[t]{5}{*}{ rs1052555 } & G & 966 & 385 & 1 & & 326 & 1 & \\
\hline & A & 54 & 35 & $1.63(1.05-2.53)$ & 0.029 & 22 & $1.21(0.72-2.01)$ & 0.470 \\
\hline & GG & 457 & 175 & 1 & & 152 & 1 & \\
\hline & GA & 52 & 35 & $1.75(1.10-2.79)$ & 0.019 & 22 & $1.28(0.74-2.20)$ & 0.375 \\
\hline & $\mathrm{AA}$ & 1 & 0 & - & - & 0 & - & - \\
\hline \multirow{4}{*}{ rs238406 } & GG & 152 & 69 & 1 & & 69 & 1 & \\
\hline & GT & 246 & 94 & $0.82(0.56-1.19)$ & 0.297 & 71 & $0.63(0.43-0.94)$ & 0.024 \\
\hline & TT & 112 & 48 & $0.93(0.60-1.46)$ & 0.763 & 34 & $0.66(0.41-1.08)$ & 0.099 \\
\hline & GT-TT & 358 & 142 & $0.86(0.60-1.21)$ & 0.378 & 105 & $0.64(0.45-0.93)$ & 0.018 \\
\hline
\end{tabular}

NSCLC, non-small cell lung cancer; SNP, single-nucleotide polymorphism; OR, odds ratio; CI, confidence interval. $p$ values were calculated by logistic regression analysis with adjustments for age and gender. Bold $p<0.05$ means the data are statistically significant. “_" represents the $p$ values were unavailable due to the absence of frequency of genotype. 
Table 5. Relationships between $X P D$ and $X P F$ polymorphisms and lymph nodes metastasis in NSCLC patients

\begin{tabular}{|c|c|c|c|c|c|c|}
\hline SNP id & Model & $\begin{array}{l}\text { Allele/ } \\
\text { genotype }\end{array}$ & $\begin{array}{l}\text { Metastasis } \\
\text { group }\end{array}$ & $\begin{array}{l}\text { Nonmetastasis } \\
\text { group }\end{array}$ & OR (95\% CI) & $p$ value \\
\hline \multirow[t]{10}{*}{ rs238406 } & \multirow[t]{3}{*}{ Allele } & G & 306 & 126 & 1 & \multirow{3}{*}{0.289} \\
\hline & & $\mathrm{T}$ & 232 & 80 & $1.19(0.86-1.66)$ & \\
\hline & & GG & 90 & 45 & 1 & \\
\hline & Heterozygote & GT & 126 & 36 & $1.72(1.03-2.89)$ & 0.040 \\
\hline & Homozygote & $\mathrm{TT}$ & 53 & 22 & $1.19(0.64-2.20)$ & 0.583 \\
\hline & \multirow[t]{2}{*}{ Dominant } & GG & 90 & 45 & 1 & \multirow[b]{2}{*}{0.080} \\
\hline & & GT-TT & 179 & 58 & $1.52(0.95-2.42)$ & \\
\hline & \multirow[t]{2}{*}{ Recessive } & GG-GT & 216 & 81 & 1 & \multirow[b]{2}{*}{0.707} \\
\hline & & TT & 53 & 22 & $0.90(0.51-1.57)$ & \\
\hline & \multicolumn{2}{|l|}{ Log-additive } & & & $1.16(0.85-1.59)$ & 0.347 \\
\hline \multirow[t]{10}{*}{ rs6498486 } & \multirow[t]{3}{*}{ Allele } & $\mathrm{A}$ & 402 & 154 & 1 & \multirow{3}{*}{0.992} \\
\hline & & $\mathrm{C}$ & 136 & 52 & $1.00(0.69-1.45)$ & \\
\hline & & $\mathrm{AA}$ & 144 & 62 & 1 & \\
\hline & Heterozygote & $\mathrm{AC}$ & 114 & 30 & $1.63(0.99-2.69)$ & 0.057 \\
\hline & Homozygote & $\mathrm{CC}$ & 11 & 11 & $0.44(0.18-1.06)$ & 0.067 \\
\hline & \multirow[t]{2}{*}{ Dominant } & $\mathrm{AA}$ & 144 & 62 & 1 & \multirow[b]{2}{*}{0.254} \\
\hline & & $\mathrm{AC}-\mathrm{CC}$ & 125 & 41 & $1.31(0.82-2.08)$ & \\
\hline & \multirow[t]{2}{*}{ Recessive } & $\mathrm{AA}-\mathrm{AC}$ & 258 & 92 & 1 & \\
\hline & & $\mathrm{CC}$ & 11 & 11 & $0.36(0.15-0.86)$ & 0.022 \\
\hline & Log-additive & & & & $1.00(0.69-1.46)$ & 0.993 \\
\hline
\end{tabular}

NSCLC, non-small cell lung cancer; SNP, single-nucleotide polymorphism; OR, odds ratio; CI, confidence interval. $p$ values were calculated by logistic regression analysis with adjustments for age and gender. Bold $p<$ 0.05 means the data are statistically significant.

lung adenocarcinoma. In squamous cell carcinoma, rs 238406 had a decreased risk (T vs. $\mathrm{G}, \mathrm{OR}=0.78,95 \% \mathrm{CI}$ : $0.61-1.00, p=0.047$; GT vs. GG, OR $=0.63,95 \%$ CI: $0.43-$ $0.94, p=0.024$; and GT-TT vs. GG, OR $=0.64,95 \% \mathrm{CI}$ : $0.45-0.93, p=0.018)$. We did not find the significant difference in the $X P D$ and $X P F$ genotypes at different stages of NSCLC $(p>0.05)$. We found that rs238406 GT genotype $(\mathrm{OR}=1.72,95 \%$ CI: $1.03-2.89, p=0.040)$ was highly presented in patients with metastasis, while rs6498486 CC genotype (OR $=0.36,95 \%$ CI: $0.15-0.86, p=0.022)$ was highly presented in patients without metastasis (Table 5).

\section{Relationship of XPD and XPF Single-Nucleotide Polymorphisms with Cisplatin-Based Chemotherapy \\ Response}

Subsequently, we also evaluated the association between XPD and XPF SNPs and the toxicity or treatment response of NSCLC patients with cisplatin-based chemo- therapy (Table 6). Among the 189 patients with cisplatinbased chemotherapy, 37 (19.6\%) patients suffered from toxicity and $42(22.2 \%)$ patients were chemotherapy responders (CR + PR). In the cisplatin-related toxicity analysis, rs231127 CC genotype was conferred to an increased risk of toxicity compared with TT-TC genotype (OR = $4.15,95 \%$ CI: $1.44-11.93, p=0.008)$. In the respond of cisplatin therapy, rs13181-G allele and TG genotype were associated with cisplatin-based chemotherapy response under allele $(\mathrm{OR}=2.37,95 \% \mathrm{CI}: 1.12-5.01, p=0.021)$, heterozygote $(\mathrm{OR}=2.73,95 \% \mathrm{CI}: 1.11-6.73, p=0.029)$, dominant $(\mathrm{OR}=2.92,95 \% \mathrm{CI}: 1.21-7.07, p=0.017)$, and additive ( $\mathrm{OR}=2.91,95 \%$ CI: $1.23-6.86, p=0.015)$ models. The rs 1052555 variants were closely associated with the effectiveness of cisplatin therapy on NSCLC under allele (OR $=2.67,95 \% \mathrm{CI}: 1.12-6.36, p=0.022)$, dominant $(\mathrm{OR}=2.89,95 \% \mathrm{CI}: 1.07-7.86, p=0.037)$, and additive $(\mathrm{OR}=2.88,95 \% \mathrm{CI}: 1.1-7.54, p=0.031)$ models. 
Table 6. Relationships between $X P D$ and $X P F$ polymorphisms and cisplatin-based chemotherapy response in NSCLC patients

\begin{tabular}{|c|c|c|c|c|}
\hline SNP id & Model & Genotype & OR (95\% CI) & $p$ value \\
\hline \multicolumn{5}{|c|}{ Toxicity group versus nontoxicity group } \\
\hline \multirow[t]{6}{*}{ rs 231127} & Allele & $\mathrm{C}$ & $1.24(0.73-2.12)$ & 0.424 \\
\hline & Heterozygote & TC & $0.43(0.18-1.02)$ & 0.056 \\
\hline & Homozygote & $\mathrm{CC}$ & $2.79(0.91-8.55)$ & 0.072 \\
\hline & Dominant & TC-CC & $0.72(0.34-1.51)$ & 0.383 \\
\hline & Recessive & $\mathrm{CC}$ & $4.15(1.44-11.93)$ & 0.008 \\
\hline & Log-additive & & $1.22(0.71-2.1)$ & 0.470 \\
\hline \multicolumn{5}{|c|}{$C R+P R$ group versus $S D+P D$ group } \\
\hline \multirow[t]{6}{*}{ rs13181 } & Allele & G & $2.37(1.12-5.01)$ & 0.021 \\
\hline & Heterozygote & TG & $2.73(1.11-6.73)$ & 0.029 \\
\hline & Homozygote & GG & - & - \\
\hline & Dominant & TG-GG & $2.92(1.21-7.07)$ & 0.017 \\
\hline & Recessive & GG & - & - \\
\hline & Log-additive & & $2.91(1.23-6.86)$ & 0.015 \\
\hline \multirow[t]{6}{*}{ rs1052555 } & Allele & $\mathrm{A}$ & $2.67(1.12-6.36)$ & 0.022 \\
\hline & Heterozygote & GA & $2.65(0.94-7.42)$ & 0.064 \\
\hline & Homozygote & AA & - & - \\
\hline & Dominant & GA-AA & $2.89(1.07-7.86)$ & 0.037 \\
\hline & & $\mathrm{AA}$ & - & - \\
\hline & Log-additive & & $2.88(1.1-7.54)$ & 0.031 \\
\hline
\end{tabular}

NSCLC, non-small cell lung cancer; SNP, single-nucleotide polymorphism; OR, odds ratio; CI, confidence interval; CR, complete response; $\mathrm{PR}$, partial response; SD, stable disease; $\mathrm{PD}$, progression of disease. $p$ values were calculated by logistic regression analysis with adjustments for age and gender. Bold $p<0.05$ means the data are statistically significant. “-” represents the $p$ values were unavailable due to the absence of frequency of genotype.

\section{Discussion}

Here, we evaluated relationships of 8 SNPs in XPD/ $X P F$ with the susceptibility and cisplatin-based chemotherapeutic response to NSCLC among a Chinese Han population in high-altitude areas. Our study found XPD rs13181 and rs1052555 possibly contributed to the increased susceptibility of lung adenocarcinoma, while $X P D$ rs238406 was a protective factor for lung squamous cell carcinoma. In addition, we also displayed that age, gender, BMI, smoking, and drinking might affect the correlation between $X P D$ and $X P F$ variants and NSCLC risk. More importantly, $X P D$ rs13181, XPD rs1052555, and $X P F$ rs231127 were associated with cisplatin-based chemotherapy response of NSCLC patients.
XPD gene, mapped on chromosome 19q13.3, plays an important role in the NER pathway, which is involved in transcription-coupled NER. XPD polymorphisms can alter the secondary structure of mRNA [18]. Several studies have found some SNPs of XPD may be related to the susceptibility of NSCLC and the toxicity of NSCLC patients receiving chemotherapy $[13,19]$. Rs13181 (T>G, Lys$751 \mathrm{Gln}$ ) is a nonsynonymous SNP in XPD, and the G allele has the capability to reduce the efficiency of DNA repair [20]. Therefore, the $\mathrm{G}$ allele of rs 13181 might be a possible risk factor for NSCLC. Although several studies showed that rs13181 was related to NSCLC risk, there were inconsistencies in the Chinese populations. For example, Liu et al. [13] suggested that the G allele of rs13181 was associated with increased susceptibility of NSCLC, 
while Qian et al. [14] observed no significant association between rs13181 and NSCLC risk. Such inconsistencies in these reports might be attributable to different environments or insufficient sample sizes. In our study, we found that rs13181-G allele increased 1.53-fold risk of lung adenocarcinoma. Rs13181 variant was also related to an increased risk of NSCLC in the subgroup at age $>59$ years or in drinkers. Additionally, G allele of rs13181 presented a better response to cisplatin-based chemotherapy, which was inconsistent with the study in the Chinese Han population by $\mathrm{Du}$ et al. [21], but consistent with the previously published meta-analysis studies [22,23]. There were inconsistencies the inconsistent result was also found in XPD rs1052555 polymorphism. XPD rs1052555C $>\mathrm{T}$ (Asp711Asp) was not associated with response to chemotherapy of patients with NSCLC [24]. However, a meta-analysis study revealed that $\mathrm{T}$ allele of rs1052555 was a risk allele and might be significantly associated with unfavorable clinical prognosis including treatment response [19]. Consistently, rs 1052555 was also associated with cisplatin-based chemotherapy response in our study. Moreover, rs 1052555 conferred to an increased susceptibility of lung adenocarcinoma. Rs1052555 also had a higher NSCLC risk among subjects with age $>59$ years. Rs238406 (G>T, Arg156Arg) is located in the synonymous coding region, which may have a possible impact on the structure of XPD protein or the stability of mRNA [25]. Han et al. [26] revealed that rs 238406 could confer increased susceptibility to lung adenocarcinoma among the Chinese population. However, we found that rs238406 might be a protective factor for lung squamous cell carcinoma. This result requires a larger sample to validate. In addition, rs238406 was related to the decreased risk of NSCLC in males and drinkers, while increased the risk in smokers. In addition, rs238406 was also related to lymph nodes metastasis in NSCLC patients.

$\mathrm{XPF}$, located on chromosome $16 \mathrm{p} 13.12$, is also another important protein in the NER pathway, which plays a key role in DNA repair and maintenance of chromosome stability [27]. XPF polymorphisms can alter the function of $X P F$, leading to tumorigenesis and affecting the outcome of cisplatin-based chemotherapy [28]. Previously, $X P F$ variants were reported to be associated with various tumors, including colorectal, ovarian, and lung cancer [29-31]. However, few studies have focused on the association between rs231127, rs8054265, rs6498486, and rs3743538 in XPF and NSCLC susceptibility. Here, our results revealed that rs231127 and rs6498486 were associated with NSCLC risk in the subgroup with $\leq 24 \mathrm{~kg} / \mathrm{m}^{2}$, while rs3743538 contributed to NSCLC risk among subjects with $>24 \mathrm{~kg} / \mathrm{m}^{2}$. Our results also showed that rs6498486 might be related to the lower incidence of lymph node metastasis. In addition, XPF rs 231127 had a significant association with the toxicity of cisplatin-based chemotherapy. These results implied that XPF polymorphisms could influence the susceptibility and toxicity of chemotherapy to NSCLC.

Some intrinsic limitations should be considered in our study. First, the possibility of selection biases could not be completely excluded in this hospital-based case-control study. Second, the number of patients with cisplatinbased chemotherapy was relatively small. Our results should be interpreted with caution and require further validation in future studies.

\section{Conclusion}

In summary, the present study provided some cumulative evidence that $X P D$ and $X P F$ variants were related to the risk of NSCLC and modulated the toxicity of cisplatin-based chemotherapy among the Chinese Han population in high-altitude areas.

\section{Acknowledgments}

We are grateful to the individuals who participated in this study. We also thank the clinicians and hospital staff who contributed to the sample and data collection for this study.

\section{Statement of Ethics and Consent to Participate}

This study was approved by the Ethics Committee of the Qinghai Province Cancer Hospital (QHWY-LL2017-0005) and was in accordance with the Declaration of Helsinki. All participants were aware of the research protocol and signed informed consent form.

\section{Conflict of Interest Statement}

The authors have no actual or potential conflicts of interest related to this manuscript. Appropriate approval was obtained, and appropriate procedures were followed concerning human subjects.

\section{Funding Sources}

This work was supported by the fund of Qinghai provincial science and technology department (2017-ZJ-707). 


\section{Author Contributions}

The work presented here was carried out in collaboration between all authors. Miao Li carried out the molecular genetic studies and drafted the manuscript. Rong Chen collected clinical information about patients and designed primers. Baoyan Ji and Chunmei Fan performed the SNP genotyping experiments. Guanying Wang and Chenli Yue performed the statistical analyses and interpreted the results. Guoquan Li conceived of the study, worked on associated data collection and their interpretation, participated in the design and coordination of the study, and funded the study. All authors read and approved the final manuscript.

\section{Availability of Data and Material}

All the data regarding the findings are available within the manuscript. Anyone who is interested in the information should contact the corresponding author.

\section{References}

1 Bray F, Ferlay J, Soerjomataram I, Siegel RL, Torre LA, Jemal A. Global cancer statistics 2018: GLOBOCAN estimates of incidence and mortality worldwide for 36 cancers in 185 countries. CA Cancer J Clin. 2018;68(6):394424.

2 Chen W, Zheng R, Baade PD, Zhang S, Zeng $\mathrm{H}$, Bray $\mathrm{F}$, et al. Cancer statistics in China, 2015. CA Cancer J Clin. 2016;66(2):115-32.

3 Malhotra J, Malvezzi M, Negri E, La Vecchia C, Boffetta P. Risk factors for lung cancer worldwide. Eur Respir J. 2016;48(3):889-902.

4 Huang CY, Xun XJ, Wang AJ, Gao Y, Ma JY, Chen YT, et al. CHRNA5 polymorphisms and risk of lung cancer in Chinese Han smokers. Am J Cancer Res. 2015;5(10):3241-8.

5 Xun X, Wang H, Yang H, Wang H, Wang B, Kang L, et al. CLPTM1L genetic polymorphisms and interaction with smoking and alcohol drinking in lung cancer risk: a case-control study in the Han population from northwest China. Medicine. 2014;93(28):e289.

6 Kilari D, Guancial E, Kim ES. Role of copper transporters in platinum resistance. World J Clin Oncol. 2016;7(1):106-13.

7 Wang T, Chen T, Thakur A, Liang Y, Gao L, Zhang S, et al. Association of PSMA4 polymorphisms with lung cancer susceptibility and response to cisplatin-based chemotherapy in a Chinese Han population. Clin Transl Oncol. 2015;17(7):564-9.

8 Zhang S, Thakur A, Liang Y, Wang T, Gao L, Yang T, et al. Polymorphisms in C-reactive protein and Glypican-5 are associated with lung cancer risk and Gartrokine- 1 influences Cisplatin-based chemotherapy response in a Chinese Han population. Dis Markers. 2015; 2015:824304.

9 Sancar A. DNA repair in humans. Annu Rev Genet. 1995;29:69-105.

10 Schaeffer L, Moncollin V, Roy R, Staub A, Mezzina M, Sarasin A, et al. The ERCC2/DNA repair protein is associated with the class II BTF2/TFIIH transcription factor. EMBO J. 1994;13(10):2388-92.

11 Kornguth DG, Garden AS, Zheng Y, Dahlstrom KR, Wei Q, Sturgis EM. Gastrostomy in oropharyngeal cancer patients with ERCC4 (XPF) germline variants. Int J Radiat Oncol Biol Phys. 2005;62(3):665-71.

12 Saviozzi S, Ceppi P, Novello S, Ghio P, Lo Iacono M, Borasio P, et al., Non-small cell lung can- cer exhibits transcript overexpression of genes associated with homologous recombination and DNA replication pathways. Cancer Res. 2009;69(8):3390-6.

13 Liu HX, Li J, Ye BG. Correlation between gene polymorphisms of CYP1A1, GSTP1, ERCC2, XRCC1, and XRCC3 and susceptibility to lung cancer. Genet Mol Res. 2016;15(4).

14 Qian B, Zhang H, Zhang L, Zhou X, Yu H, Chen K. Association of genetic polymorphisms in DNA repair pathway genes with non-small cell lung cancer risk. Lung Cancer. 2011;73(2):138-46.

15 Carbone DP, Minna JD. Chemotherapy for non-small cell lung cancer. BMJ. 1995; 311(7010):889-90.

16 Eisenhauer EA, Therasse P, Bogaerts J, Schwartz LH, Sargent D, Ford R, et al. New response evaluation criteria in solid tumours: revised RECIST guideline (version 1.1). Eur J Cancer. 2009;45(2):228-47.

17 Wang LE, Gorlova OY, Ying J, Qiao Y, Weng $\mathrm{SF}$, Lee AT, et al. Genome-wide association study reveals novel genetic determinants of DNA repair capacity in lung cancer. Cancer Res. 2013;73(1):256-64.

18 Wolfe KJ, Wickliffe JK, Hill CE, Paolini M, Ammenheuser MM, Abdel-Rahman SZ. Single nucleotide polymorphisms of the DNA repair gene XPD/ERCC2 alter mRNA expression. Pharmacogenet Genomics. 2007;17(11): 897-905.

19 Tan LM, Qiu CF, Zhu T, Jin YX, Li X, Yin JY, et al. Genetic polymorphisms and platinumbased chemotherapy treatment outcomes in patients with non-small cell lung cancer: a genetic epidemiology study based meta-analysis. Sci Rep. 2017;7(1):5593.

20 Benhamou S, Sarasin A. ERCC2/XPD gene polymorphisms and lung cancer: a HuGE review. Am J Epidemiol. 2005;161(1):1-14.

21 Du Y, Su T, Zhao L, Tan X, Chang W, Zhang $\mathrm{H}$, et al. Associations of polymorphisms in DNA repair genes and MDR1 gene with chemotherapy response and survival of non-small cell lung cancer. PLoS One. 2014;9(6):e99843.

22 Wang J, Liu Q, Yuan S, Xie W, Liu Y, Xiang Y, et al. Genetic predisposition to lung cancer: comprehensive literature integration, metaanalysis, and multiple evidence assessment of candidate-gene association studies. Sci Rep. 2017;7(1):8371.
23 Yu SN, Liu GF, Li XF, Fu BH, Dong LX, Zhang $\mathrm{SH}$. Evaluation of prediction of polymorphisms of DNA repair genes on the efficacy of platinum-based chemotherapy in patients with non-small cell lung cancer: a network meta-analysis. J Cell Biochem. 2017;118(12): 4782-91.

24 Lee SY, Kang HG, Yoo SS, Kang YR, Choi YY, Lee WK, et al. Polymorphisms in DNA repair and apoptosis-related genes and clinical outcomes of patients with non-small cell lung cancer treated with first-line paclitaxel-cisplatin chemotherapy. Lung Cancer. 2013;82(2): 330-9.

25 Wu W, Zhang W, Qiao R, Chen D, Wang H, Wang Y, et al. Association of XPD polymorphisms with severe toxicity in non-small cell lung cancer patients in a Chinese population. Clin Cancer Res. 2009;15(11):3889-95.

26 Han L, Lee C-K, Pang H, Chan H-T, Lo I-L, Lam S-K, et al. Genetic predisposition to lung adenocarcinoma among never-smoking Chinese with different epidermal growth factor receptor mutation status. Lung Cancer. 2017; 114:79-89.

27 Manandhar M, Boulware KS, Wood RD. The ERCC1 and ERCC4 (XPF) genes and gene products. Gene. 2015;569(2):153-61.

28 Zhang R, Jia M, Xu Y, Qian D, Wang M, Zhu $\mathrm{M}$, et al. An ERCC4 regulatory variant predicts grade- 3 or -4 toxicities in patients with advanced non-small cell lung cancer treated by platinum-based therapy. Int J Cancer. 2018; 142(6):1218-29.

29 Dziki L, Dziki A, Mik M, Majsterek I, Kabzinski J. Modulation of colorectal cancer risk by polymorphisms in $51 \mathrm{Gln} / \mathrm{His}$, 64Ile/Val, and 148Asp/Glu of APEX gene; 23Gly/Ala of XPA gene; and 689Ser/Arg of ERCC4 gene. Gastroenterol Res Pract. 2017;2017: 3840243.

30 Zhao Z, Zhang A, Zhao Y, Xiang J, Yu D, Liang $Z$, et al. The association of polymorphisms in nucleotide excision repair genes with ovarian cancer susceptibility. Biosci Rep. 2018;38(3): BSR20180114.

31 Sullivan I, Salazar J, Majem M, Pallarés C, Del Río E, Páez D, et al. Pharmacogenetics of the DNA repair pathways in advanced non-small cell lung cancer patients treated with platinum-based chemotherapy. Cancer Lett. 2014; 353(2):160-6. 